\title{
Claudin-4 expression distinguishes SWI/SNF complex-deficient undifferentiated carcinomas from sarcomas
}

\author{
Inga-Marie Schaefer ${ }^{1}$, Abbas Agaimy ${ }^{2}$, Christopher DM Fletcher ${ }^{1}$ and Jason L Hornick ${ }^{1}$ \\ ${ }^{1}$ Department of Pathology, Brigham and Women's Hospital, Harvard Medical School, Boston, MA, USA and \\ ${ }^{2}$ Institute of Pathology, Friedrich-Alexander University Erlangen-Nuremberg, University Hospital of Erlangen, \\ Erlangen, Germany
}

\begin{abstract}
Inactivation of SWI/SNF (switch/sucrose non-fermentable) chromatin remodeling complex subunits is a feature shared by select carcinomas and sarcomas with epithelioid morphology and variable keratin expression, making the distinction between carcinoma and sarcoma challenging in some cases. The tight junction-associated protein claudin-4 is a marker of epithelial differentiation that is expressed in nearly all carcinomas. Claudin-4 expression has been reported in the glandular component of biphasic synovial sarcoma but has not been systematically evaluated in other sarcoma types. In this study we assessed claudin-4 expression in SWI/SNF complex-deficient neoplasms showing loss of SMARCB1 (INI1), SMARCA4 (BRG1), or ARID1A and other sarcomas with epithelioid morphology. Immunohistochemistry for claudin-4 was performed on 130 neoplasms, including 90 soft tissue tumors with epithelioid morphology and/or SMARCB1 deficiency (20 epithelioid sarcomas (10 conventional, 10 proximal-type); 10 epithelioid angiosarcomas; 10 epithelioid hemangioendotheliomas; 15 epithelioid malignant peripheral nerve sheath tumors; 10 malignant rhabdoid tumors; 15 myoepithelial carcinomas; 10 biphasic synovial sarcomas), 10 ovarian clear cell carcinomas, 10 ovarian small cell carcinomas of hypercalcemic type, and 20 SWI/SNF complex-deficient undifferentiated carcinomas (14 SMARCB1 deficient and 6 SMARCA4 deficient, including rhabdoid carcinomas of various sites and sinonasal carcinomas). Membranous expression of claudin- 4 ( $\geq 5 \%$ of cells) was observed in all biphasic synovial sarcomas (epithelial component only), all ovarian clear cell carcinomas, and 16 (80\%) SWI/SNF complex-deficient undifferentiated carcinomas. All other soft tissue tumors were negative for claudin-4, with the exception of two myoepithelial carcinomas and one malignant rhabdoid tumor. Interestingly, none of the ovarian small cell carcinomas of hypercalcemic type expressed claudin-4. In summary, expression of claudin-4 is highly specific for true epithelial differentiation and may be useful to distinguish SWI/SNF complex-deficient undifferentiated carcinomas from sarcomas with epithelioid morphology. The lack of claudin-4 expression in ovarian small cell carcinomas of hypercalcemic type suggests that these tumors may be better regarded as sarcomas rather than carcinomas. Modern Pathology (2017) 30, 539-548; doi:10.1038/modpathol.2016.230; published online 13 January 2017
\end{abstract}

Claudins are integral components of tight junctions which form barriers in epithelial, endothelial, and perineurial cells to separate different microanatomic compartments and allow for the selective exchange of substances between the apical and basolateral cellular domains. ${ }^{1}$ Tight junctions are also involved in signaling cascades that regulate cell growth and differentiation. Claudin-4, first described in 1999 in

Correspondence: Dr JL Hornick, MD, PhD, Department of Pathology, Brigham and Women's Hospital, 75 Francis Street, Boston, MA 02115, USA.

E-mail: jhornick@partners.org

Received 22 September 2016; revised 9 November 2016; accepted 23 November 2016; published online 13 January 2017 murine epithelial cells in the lungs and kidneys, ${ }^{2}$ has subsequently been shown to be expressed in most epithelial cells and carcinomas and has been validated as a useful marker in the distinction of mesothelioma, which usually lacks claudin-4 expression, from metastatic adenocarcinoma, which consistently shows immunoreactivity for this protein. ${ }^{3-5}$

Expression of claudin-4 has also been observed in the epithelial component of biphasic synovial sarcoma and a subset of desmoplastic small round cell tumors, whereas other spindle cell sarcomas and melanoma lack expression. ${ }^{4}$ Other tight junctionassociated proteins, such as claudin-1, ZO-1, and occludin, were previously detected in $90 \%$ of 
Table 1 Summary of immunohistochemical staining for claudin-4 in 130 neoplasms including sarcomas with epithelioid morphology and SWI/SNF complex-deficient undifferentiated carcinomas

\begin{tabular}{|c|c|c|c|}
\hline Tumor type & Total cases & Claudin-4 positive ${ }^{\mathrm{a}}$ & SWI/SNF complex deficiency \\
\hline Epithelioid sarcoma & 20 & $0(0 \%)$ & SMARCB1 (100\%) \\
\hline Epithelioid angiosarcoma & 10 & $0(0 \%)$ & - \\
\hline Epithelioid hemangioendothelioma & 10 & $0(0 \%)$ & - \\
\hline Epithelioid malignant peripheral nerve sheath tumor & 15 & $0(0 \%)$ & SMARCB1 (100\%) \\
\hline Malignant rhabdoid tumor & 10 & $1(10 \%)$ & SMARCB1 $(100 \%)$ \\
\hline Myoepithelial carcinoma & 15 & $2(13 \%)$ & SMARCB1 (13\%) \\
\hline Biphasic synovial sarcoma & 10 & $10(100 \%)^{b}$ & - \\
\hline Ovarian clear cell carcinoma & 10 & $10(100 \%)$ & ARID1A (50\%) \\
\hline Ovarian small cell carcinoma of hypercalcemic type & 10 & $0(0 \%)$ & SMARCA4 $(100 \%)$ \\
\hline SWI/SNF complex-deficient carcinomas & 20 & $16(80 \%)$ & SMARCB1 (70\%); SMARCA4 (30\%) \\
\hline
\end{tabular}

${ }^{\mathrm{a}}$ Membranous claudin-4 expression observed in $\geq 5 \%$ of tumor cells. ${ }^{\mathrm{b}}$ Only in epithelial component.
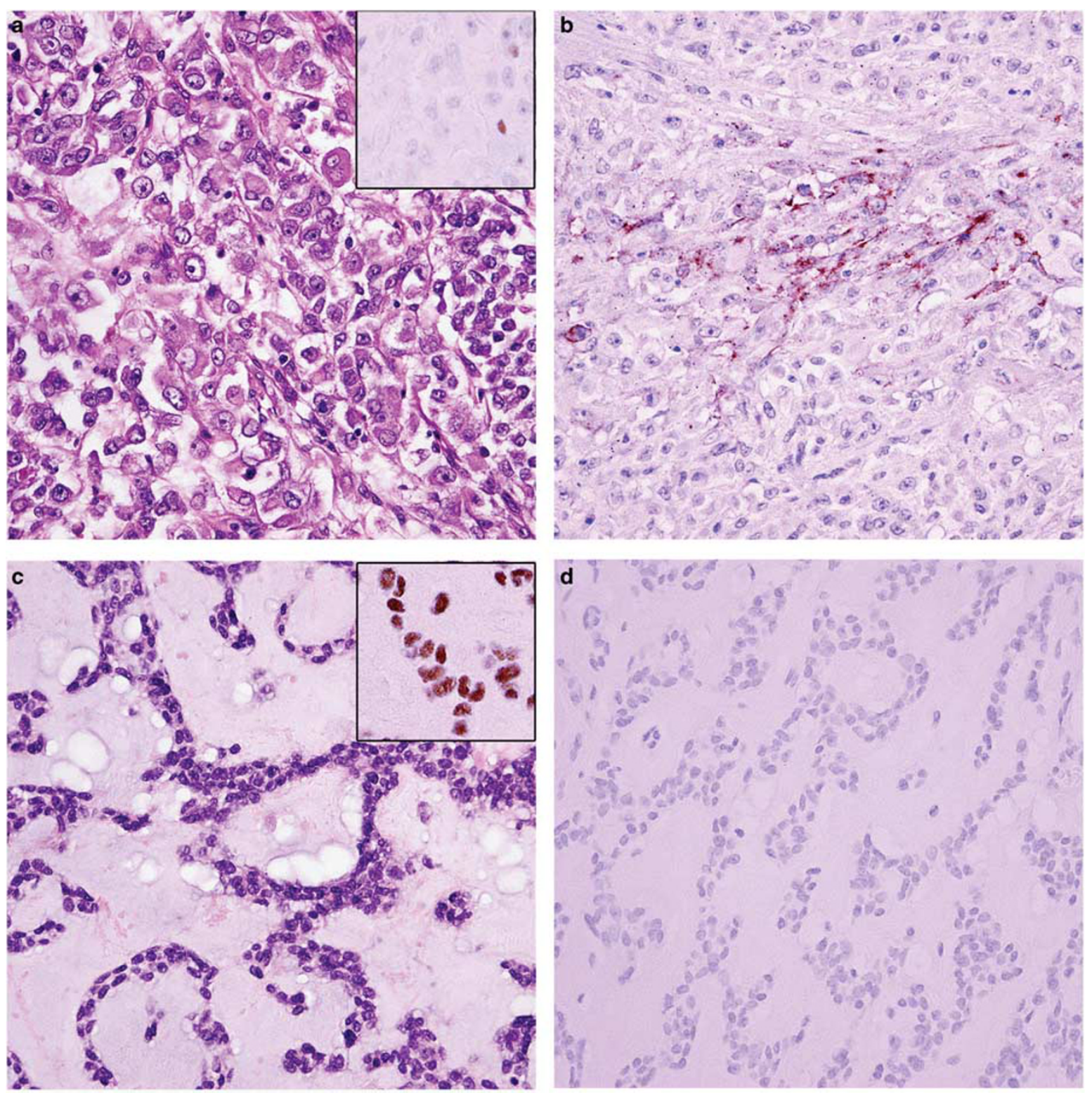

Figure 1 SMARCB1-deficient sarcomas occasionally positive for claudin-4. Malignant rhabdoid tumor (a) with loss of SMARCB1 (inset) and multifocal expression of claudin-4 (b). A myoepithelial carcinoma (c) with retained SMARCB1 expression (inset) and negative staining for claudin-4 (d), and a myoepithelial carcinoma (e) with retained SMARCB1 expression (inset) and multifocal staining for claudin-4 (f). 

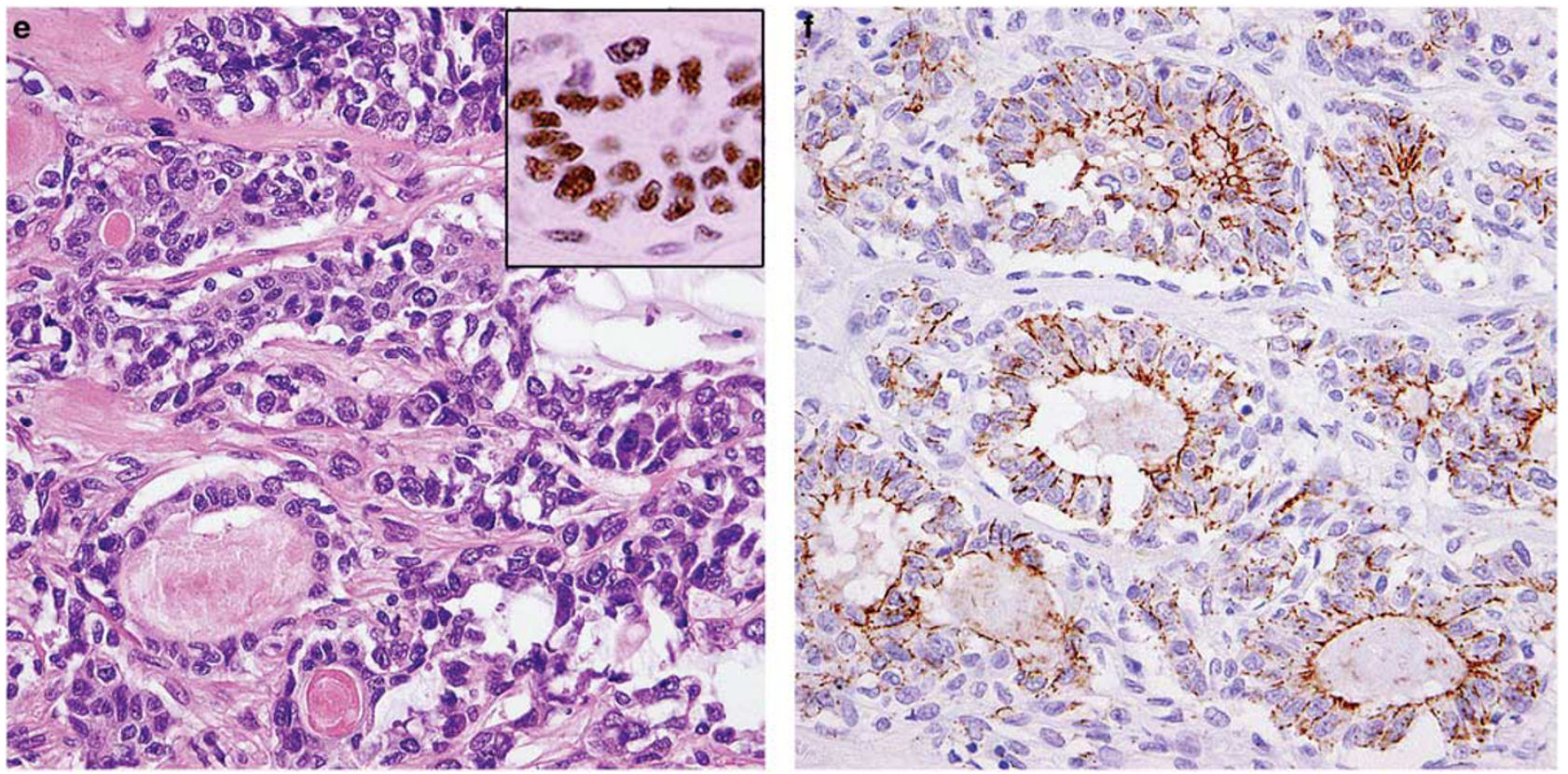

Figure 1 Continued.

Ewing sarcomas, suggesting partial epithelial differentiation. ${ }^{6}$ The same tight junction-associated proteins have been shown to be expressed in synovial sarcoma, including monophasic, biphasic, and poorly differentiated subtypes, to various extents. ${ }^{7}$ Although expression of ZO-1 and claudin-1 has been reported in a subset of malignant peripheral nerve sheath tumors and malignant solitary fibrous tumors, ${ }^{7}$ claudin-4 has not been systematically studied in sarcomas with epithelioid morphology.

The SWI/SNF (switch/sucrose non-fermentable) chromatin remodeling complex has a fundamental role in regulation of gene expression. Owing to its tumor suppressor properties, the SWI/SNF complex has been increasingly implicated in the pathogenesis of diverse neoplasms of mesenchymal and epithelial origin characterized by inactivation (deficiency) of one or more SWI/SNF complex components, including SMARCB1 (INI1), SMARCA4 (BRG1), and ARID1A. ${ }^{8}$ Malignant rhabdoid tumor and epithelioid sarcoma, as well as a subset of myoepithelial carcinomas and epithelioid malignant peripheral nerve sheath tumors, share SMARCB1 deficiency, whereas ovarian small cell carcinomas of hypercalcemic type are characterized by SMARCA4 deficiency in nearly all cases. ARID1A deficiency is observed in around half of ovarian clear cell carcinomas. In the past few years, rare SWI/SNF complex-deficient undifferentiated carcinomas have been reported at various anatomic locations, including the gastrointestinal tract, ${ }^{9}$ endometrium,,${ }^{10}$ urinary tract, ${ }^{11}$ and sinonasal tract; ${ }^{12-14}$ such tumors often show rhabdoid histologic features. There remains some dispute regarding the nosologic status of these tumors; the consensus among authors who have studied these tumor types, based in part upon the presence of conventional carcinomatous components and shared genomic findings in a subset of cases, has been that these tumors are epithelial in nature. Due to their undifferentiated morphology and inconsistent expression of lineage-specific immunohistochemical markers, diagnosis of these neoplasms may be challenging and only made after the exclusion of more common carcinomas or sarcomas with epithelioid morphology and SWI/SNF complex deficiency.

To validate its potential diagnostic utility, we evaluated immunohistochemistry for claudin-4 in sarcomas with epithelioid morphology and/or SMARCB1 deficiency and SWI/SNF complexdeficient undifferentiated carcinomas.

\section{Materials and methods}

Cases were retrieved from the surgical pathology and consult files of Brigham and Women's Hospital and University Hospital Erlangen as well as the consult files of three of the authors (AA, CDMF and JLH). In total, 130 neoplasms were studied, including 20 epithelioid sarcomas (10 conventional and 10 proximal-type, all SMARCB1 deficient), 10 epithelioid angiosarcomas, 10 epithelioid hemangioendotheliomas, 15 epithelioid malignant peripheral nerve sheath tumors (all SMARCB1 deficient), 10 malignant rhabdoid tumors (all SMARCB1 deficient), 15 myoepithelial carcinomas (two SMARCB1 deficient), 10 biphasic synovial sarcomas, 10 ovarian clear cell carcinomas (five ARID1A deficient), 10 ovarian small cell carcinomas of hypercalcemic type (all 

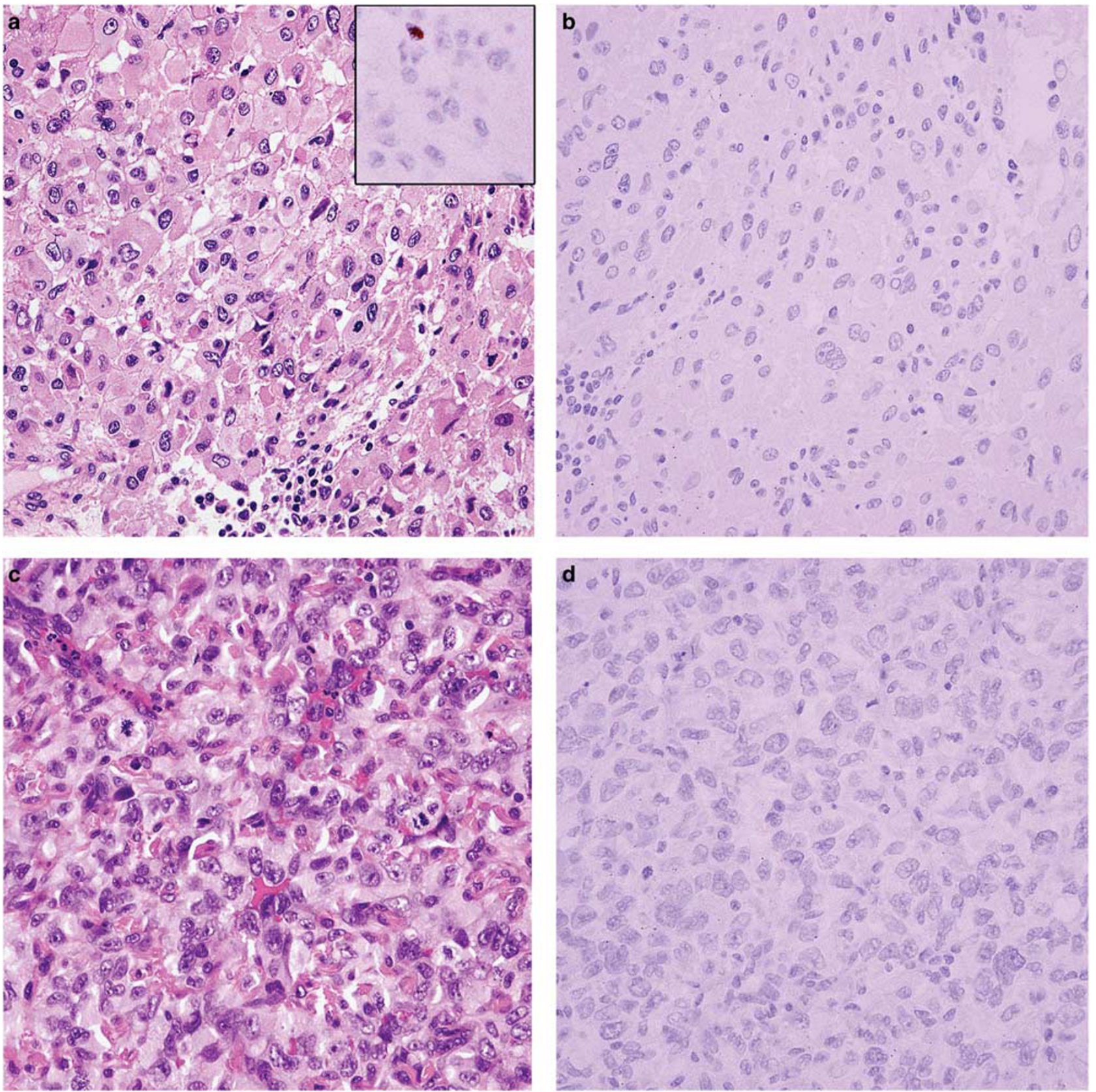

Figure 2 Sarcomas with epithelioid morphology consistently negative for claudin-4. Epithelioid sarcoma (a) with loss of SMARCB1 in tumor cells (inset; inflammatory cells serving as positive internal control) and complete absence of claudin-4 expression (b). Epithelioid angiosarcoma (c) showing lack of claudin-4 expression (d). Epithelioid malignant peripheral nerve sheath tumor (e) with loss of SMARCB1 (inset) and negative staining for claudin-4 (f).

SMARCA4 deficient), and 20 SWI/SNF complexdeficient undifferentiated carcinomas (14 SMARCB1 deficient and 6 SMARCA4 deficient). Specifically, the group of SWI/SNF complex-deficient undifferentiated carcinomas included eight SMARCB1-deficient sinonasal carcinomas, five SMARCA4-deficient rhabdoid carcinomas, five SMARCB1-deficient rhabdoid carcinomas, one SMARCB1-deficient carcinoma with glandular and sarcomatoid differentiation, and one SMARCA4-deficient dedifferentiated endometrial carcinoma; the clinical and histologic features of a subset of these tumors have been reported previously. ${ }^{9-11}$

Immunohistochemistry was performed on $4-\mu \mathrm{m}$ thick formalin-fixed paraffin-embedded whole tissue sections following pressure cooker antigen retrieval (Target Retrieval Solution; pH 6.1; Dako, Carpinteria, CA, USA) using a mouse monoclonal antibody directed against claudin-4 (1:100 dilution; clone 3E2C1; Invitrogen, Carlsbad, CA, USA). Appropriate 

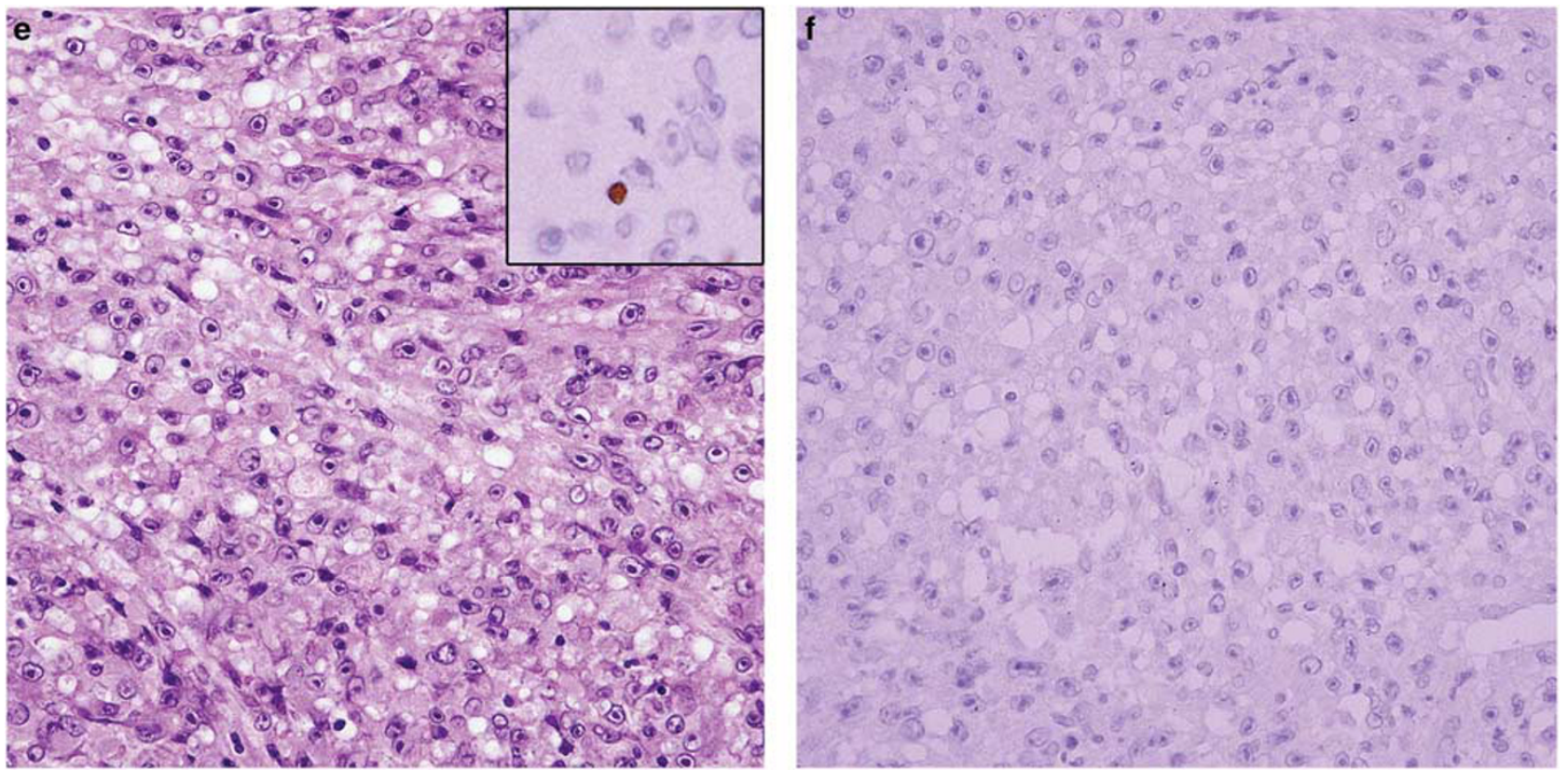

Figure 2 Continued.

positive (normal skeletal muscle, colon, and skin) and negative controls were used throughout. Extent of staining was scored as 0 (negative), $1+(<5 \%$ of cells positive), $2+(5-24 \%$ of cells positive), $3+(25-49 \%$ positive), $4+(50-74 \%$ positive), and $5+(75-100 \%$ positive). Tumors showing $2+$ or more membranous immunoreactivity were considered positive.

This study was performed with the approval of the Institutional Review Board at Brigham and Women's Hospital.

\section{Results}

The results of immunohistochemical staining for claudin-4 are shown in Table 1. Membranous expression of claudin-4 (in $\geq 5 \%$ of cells) was observed in the epithelial component only of all biphasic synovial sarcomas (10/10, 100\%), in one malignant rhabdoid tumor $(1 / 10,10 \%$; scored as $2+)$, and in two myoepithelial carcinomas $(2 / 15,13 \%$; scored as 2+ and 4+; both cases with retained SMARCB1) (Figure 1). One claudin-4-positive myoepithelial carcinoma had a focal glandular component; claudin-4 expression was observed in this component as well as in areas without glandular differentiation. The other claudin-4-positive myoepithelial carcinoma showed no histologic evidence of epithelial differentiation. Of note, one other myoepithelial carcinoma showed glandular differentiation but was negative for claudin-4. All epithelioid sarcomas, epithelioid angiosarcomas, epithelioid hemangioendotheliomas, and epithelioid malignant peripheral nerve sheath tumors were negative for claudin-4 (Figure 2). All ovarian clear cell carcinomas $(10 / 10,100 \%$; scored as $5+$ ) were diffusely positive for claudin-4, in contrast to none of the ovarian small cell carcinomas of hypercalcemic type $(0 / 10,0 \%)$ (Figure 3). Claudin-4 expression was also observed in 16/20 (80\%) SWI/SNF complexdeficient undifferentiated carcinomas (ranging from $2+$ to $5+$ ) (Figure 4). Comparing claudin-4 staining in all sarcomas with epithelioid morphology (13/90, $14 \%$ ) to SWI/SNF complex-deficient undifferentiated carcinomas $(16 / 20,80 \%)$, expression was significantly associated with true epithelial differentiation $(P<0.0001)$.

In addition to the expected membranous staining pattern, immunoreactivity for claudin-4 was also observed as a single, minute intracytoplasmic dot in 24 of $39(62 \%)$ positive cases, including $90 \%$ of synovial sarcomas, $90 \%$ of ovarian clear cell carcinomas, and $31 \%$ of SWI/SNF complex-deficient undifferentiated carcinomas (Figure 5).

\section{Discussion}

The SWI/SNF chromatin remodeling complex is a highly conserved, large multiprotein complex comprised of, to date, 15 well-characterized subunits encoded by 29 genes with a total molecular weight of $\sim 2$ MDa. ${ }^{15}$ Using energy derived from ATP hydrolysis, this 'machinery' enables compaction and decompaction of DNA to regulate accessibility to transcription factors, selective gene expression, DNA repair, and recombination. ${ }^{8,16}$ Inactivation of one or more subunit is observed in $\sim 20 \%$ of all human neoplasms. ${ }^{16}$ The patterns of genomic and functional subunit inactivation between and within different 

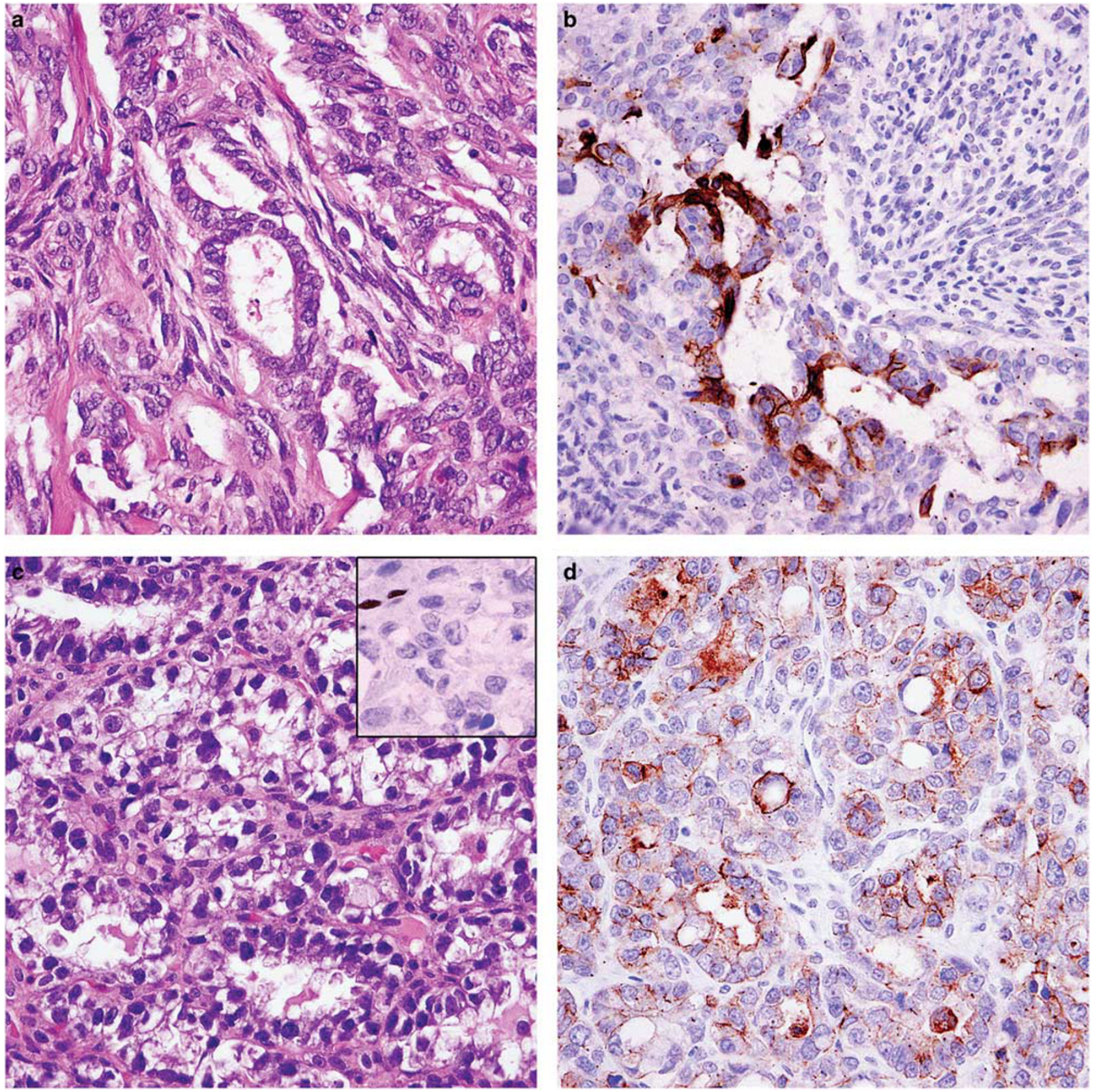

Figure 3 Biphasic synovial sarcoma (a) with membranous claudin-4 expression in the epithelial component and negative staining in the spindle cell component (b). Ovarian clear cell carcinoma (c) with loss of ARID1A (inset) and diffuse immunoreactivity for claudin-4 (d), in contrast to ovarian small cell carcinoma of hypercalcemic type (e) with loss of SMARCA4 (inset), which was completely negative for claudin-4 (f).

tumor types are complex, and even though most subunits are thought to have tumor suppressor functions, their interdependency within the complex and the biologic consequences of inactivation of one or more subunits are not yet entirely understood in the various types of SWI/SNF complex-deficient neoplasms.

However, some tumors show pathognomonic inactivation of a distinct SWI/SNF complex subunit: for instance, malignant rhabdoid tumor typically harbors inactivation of the tumor suppressor
SMARCB1 (in $98 \%$ of cases), ${ }^{17}$ resulting from SMARCB1 somatic or germline mutations or deletions. In addition, SMARCB1 deficiency has also been demonstrated in other select mesenchymal neoplasms, such as epithelioid sarcoma $(\sim 90 \%$ of cases), with SMARCB1 deletions (heterozygous or homozygous) along with miR-206, -381, and 671-5p upregulation being the most common underlying mechanism, ${ }^{18}$ epithelioid malignant peripheral nerve sheath tumor (two-thirds of cases), and myoepithelial carcinoma (10-40\%; more often in 

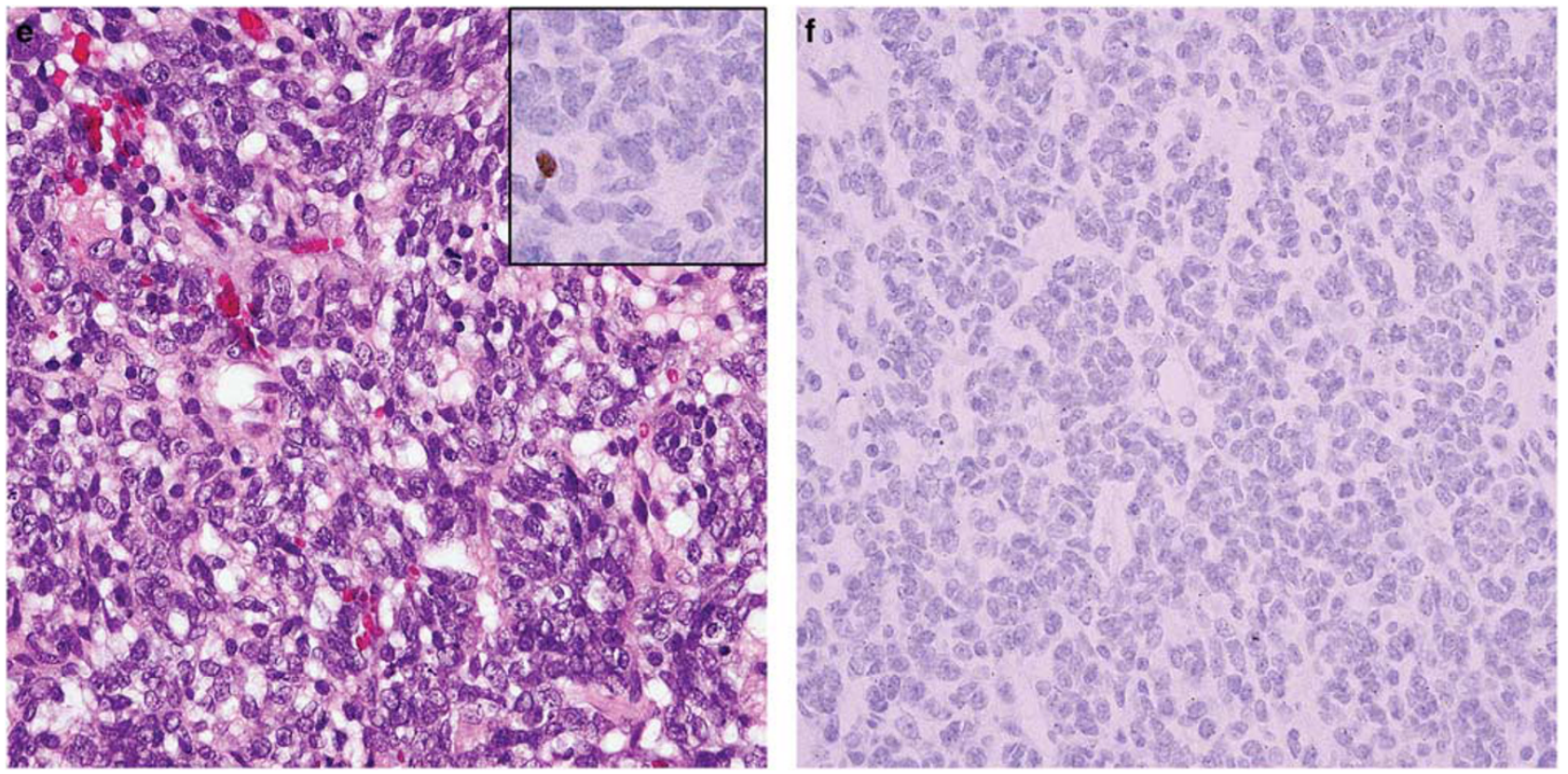

Figure 3 Continued.

pediatric cases). ${ }^{17}$ Inactivating mutations of ARID1A, another essential SWI/SNF complex subunit, have recently been demonstrated to be the driver event in approximately half of ovarian clear cell carcinomas, which show corresponding loss of ARID1A protein expression. ${ }^{19}$ Finally, ovarian small cell carcinomas of hypercalcemic type are characterized by loss of SMARCA4 and SMARCA2 expression, and underlying SMARCA4 inactivating mutations in $>90 \%$ of cases. ${ }^{20}$ In contrast to these relatively rare but well defined distinct tumor types, increasing numbers of SWI/SNF complex-deficient undifferentiated carcinomas of various anatomic site have been reported in the past few years. ${ }^{9-12}$ These tumors are characterized by aggressive clinical behavior, discohesive architecture, frequent rhabdoid morphology, and expression of keratin and/or EMA in the majority of cases.

Undifferentiated/rhabdoid carcinomas of the gastrointestinal tract have been reported to show mutually exclusive loss of SMARCA4 and SMARCA2 or co-inactivation of SMARCB1 and SMARCA2. ${ }^{9}$ In contrast, undifferentiated/dedifferentiated urothelial carcinomas have recently been shown to be SMARCA2-deficient in $\sim 70 \%$ of cases, less often characterized by loss of ARID1A, SMARCB1, SMARCA4, or SMARCC1. ${ }^{11}$ GATA3 expression is more limited in these tumors compared to conventional urothelial carcinomas. ${ }^{11}$ Recently, a rare subset of sinonasal carcinomas $(\sim 3 \%)$ has been reported to be SMARCB1 deficient. ${ }^{12}$ SMARCB1deficent sinonasal carcinomas share an infiltrative growth pattern and a basaloid to rhabdoid appearance, with tumor cells arranged in nests in a fibrous stroma. ${ }^{12}$ However, a wide range of morphologic features has been described, making their diagnosis challenging.

The distinction between these SWI/SNF complexdeficient undifferentiated carcinomas and mesenchymal neoplasms with SWI/SNF complex deficiency and epithelioid/rhabdoid morphology may be challenging. Epithelial markers such as keratin or EMA are known to be expressed not only in the majority of SWI/SNF complex-deficient carcinomas but also in a significant number of different types of sarcomas with epithelioid morphology. Furthermore, undifferentiated or rhabdoid morphology is a feature that may be shared by SWI/SNF complexdeficient carcinomas and sarcomas with epithelioid morphology.

Claudin-4 has been established as a useful marker in the distinction of carcinoma and mesothelioma. ${ }^{3-5}$ In the present study, we detected claudin-4 expression in $80 \%$ of SWI/SNF complex-deficient undifferentiated carcinomas compared with only $4 \%$ of sarcomas with epithelioid morphology, excluding biphasic synovial sarcoma. The presence of diffuse membranous claudin- 4 expression in the glandular component of all biphasic synovial sarcomas and in all ovarian clear cell carcinoma confirms its specificity for epithelial differentiation, independent of SWI/SNF complex deficiency. Based on our findings, claudin-4 may serve as a useful diagnostic adjunct in the distinction of SWI/SNF complexdeficient carcinomas from sarcomas with epithelioid morphology. The closest mesenchymal mimic is likely proximal-type epithelioid sarcoma; none of the examples included in this study showed claudin4 expression. It should be noted, however, that some such carcinomas show relatively limited staining for 

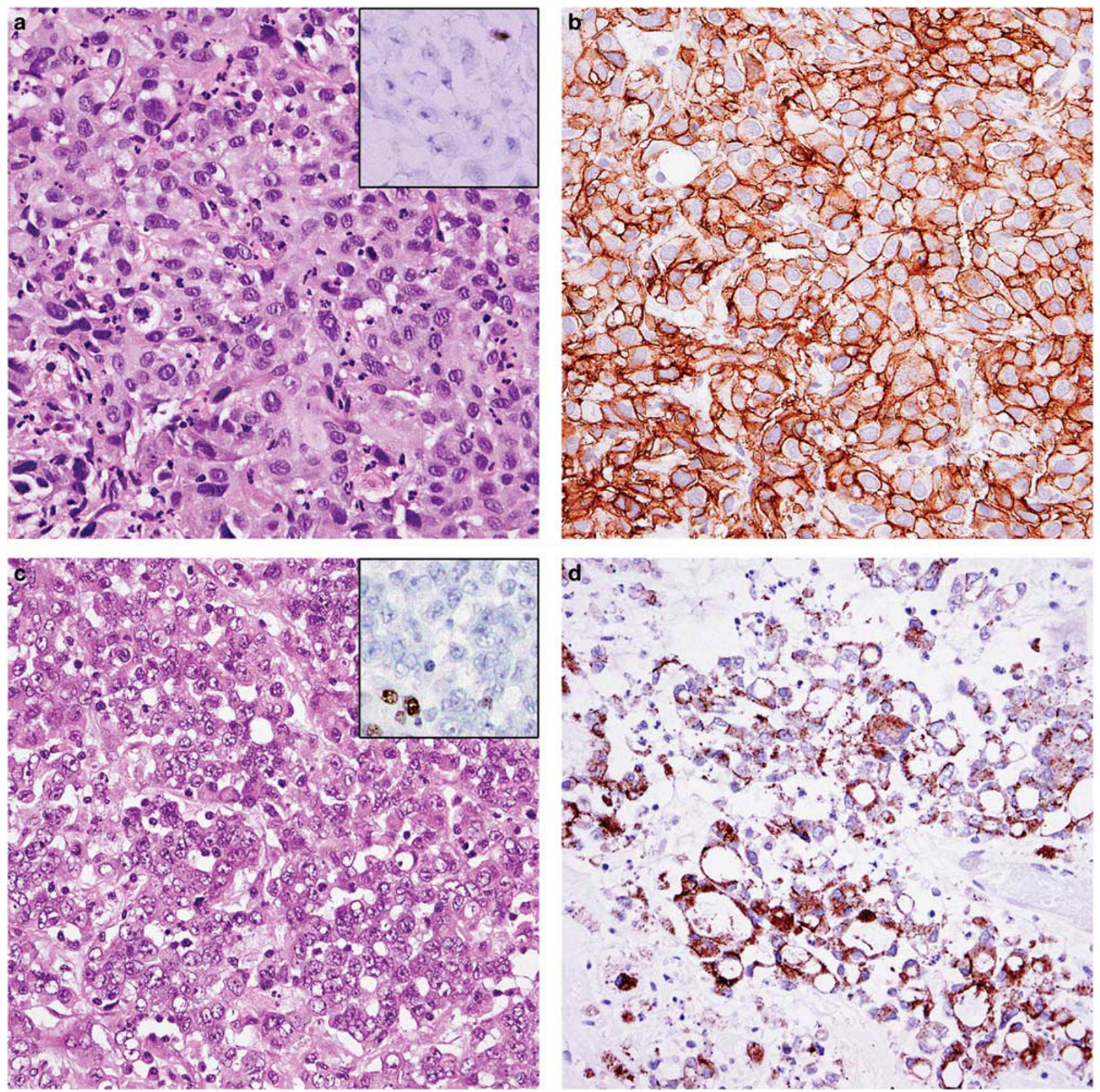

Figure 4 SWI/SNF complex-deficient undifferentiated carcinomas. Pancreatic rhabdoid carcinoma metastatic to the liver (a) with SMARCB1 loss (inset) and diffuse membranous expression of claudin-4 (b). A rhabdoid carcinoma of the cecum (c) with SMARCA4 loss (inset) and multifocal expression of claudin-4 (d). A SMARCB1-deficient sinonasal carcinoma (e) with diffuse positivity for claudin-4 (f).

claudin-4, and others completely lack claudin-4 expression; an absence of claudin-4 staining in this context therefore does not entirely exclude carcinoma.

Small cell carcinoma of hypercalcemic type is a rare ovarian tumor with a predilection for young adults and an association with hypercalcemia in $\sim 60 \%$ of cases; this tumor type follows an aggressive clinical course. ${ }^{21}$ Histologically, these tumors are characterized by small, round cells with vesicular nuclei and a high mitotic rate, which may be arranged in diffuse sheets with follicle-like space, nests, cords, or clusters. ${ }^{21}$ Until recently, due to the absence of specific immunohistochemical markers and resemblance to a wide range of other tumor types, the diagnosis of ovarian small cell carcinoma of hypercalcemic type was challenging. However, recently SMARCA4 deficiency, resulting from biallelic SMARCA4 inactivation, has been demonstrated to be highly specific and sensitive for the diagnosis of SCCOHT. ${ }^{22}$ Since claudin-4 expression seems to be restricted to neoplasms with true epithelial differentiation, the lack of expression of claudin-4 in ovarian small cell carcinoma of hypercalcemic 

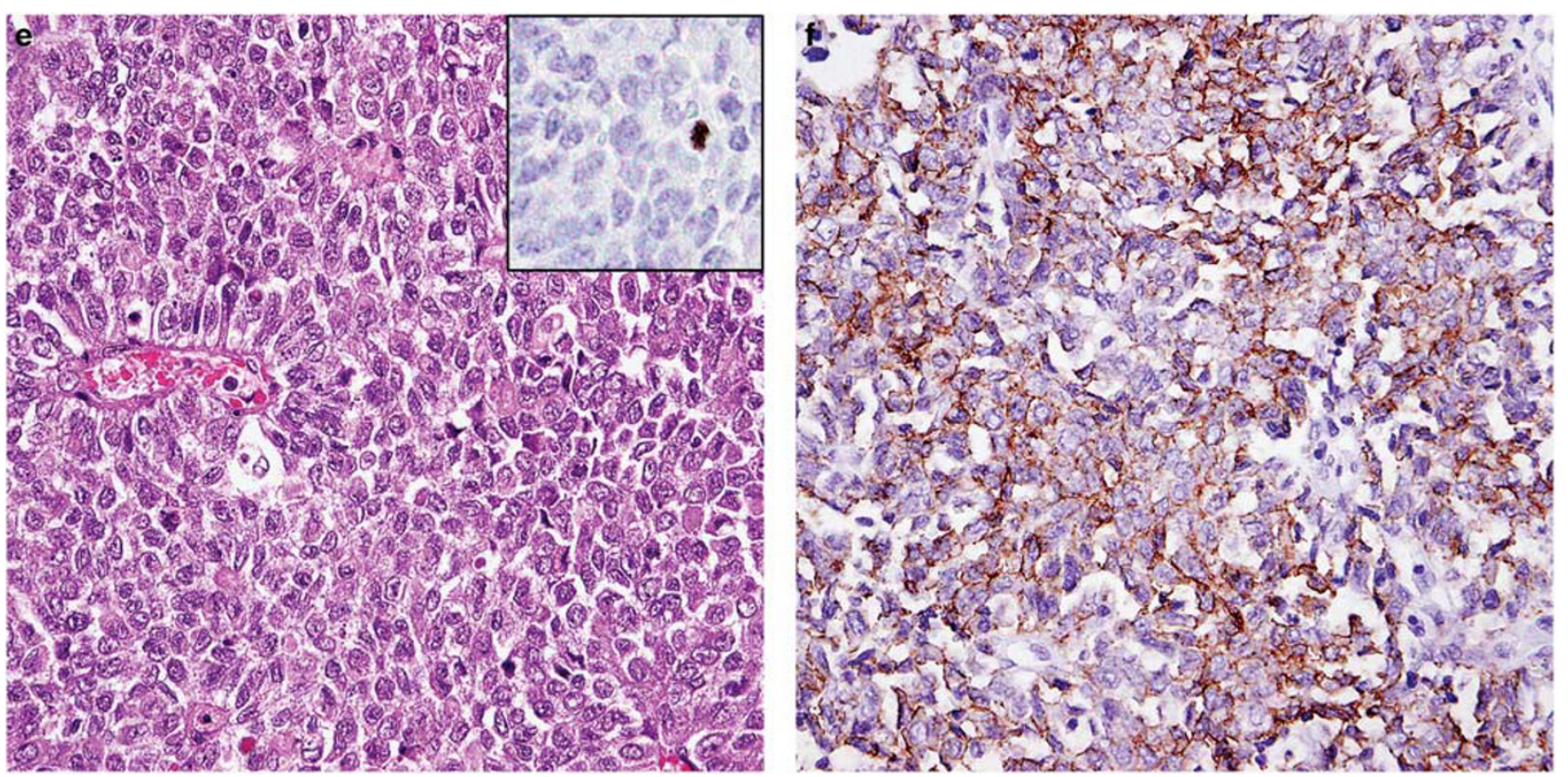

Figure 4 Continued.
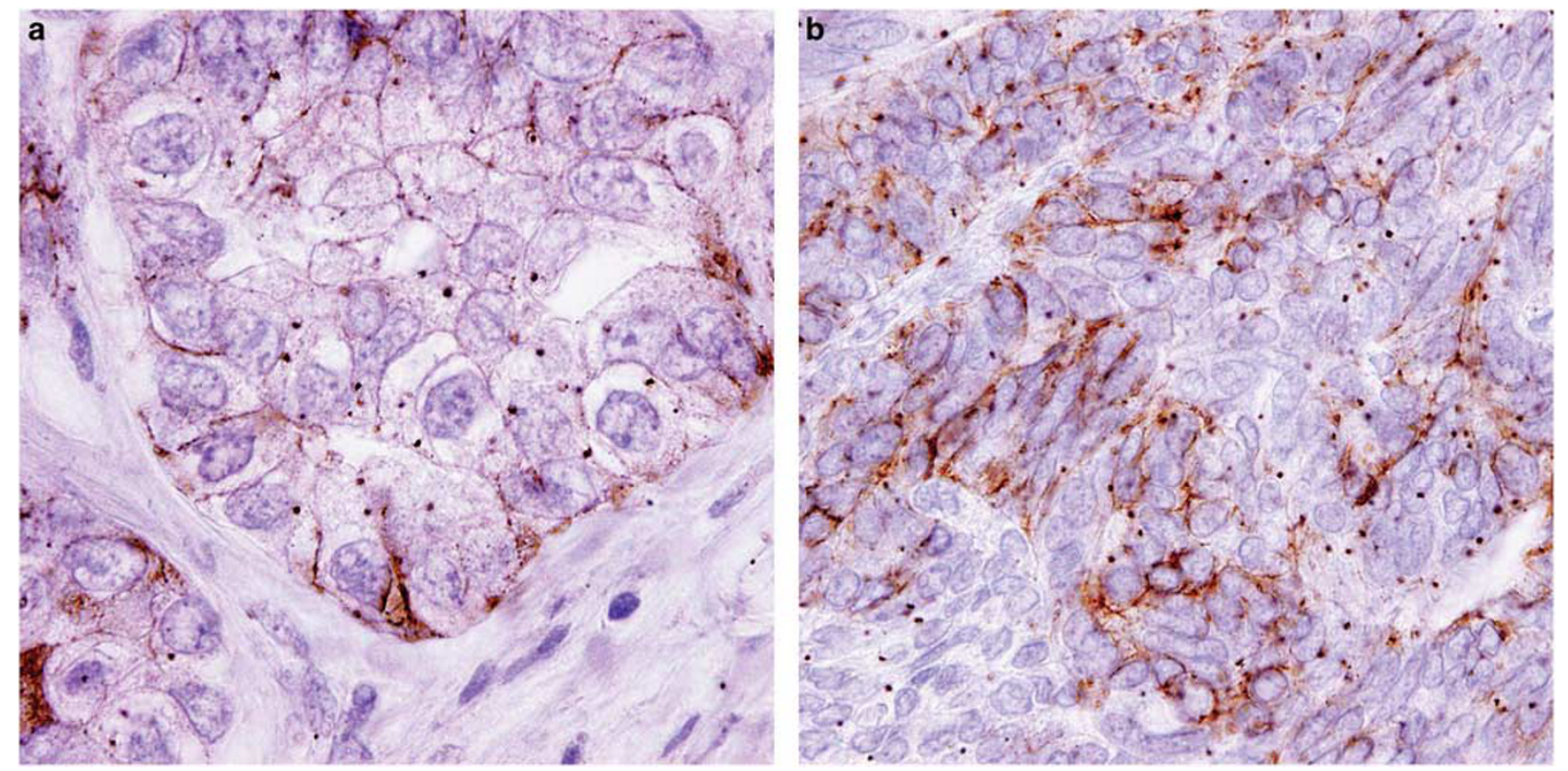

Figure 5 Some tumors with membranous claudin-4 expression, such as synovial sarcoma (a) and ovarian clear cell carcinoma (b), also showed a dot-like pattern of staining for claudin-4.

type suggests that these undifferentiated neoplasms may not be derived from an epithelial lineage.

To the best of our knowledge, the dot-like staining pattern that we observed in approximately twothirds of claudin-4-positive tumors has not been described previously. Only cases with otherwise clear membranous expression showed this peculiar pattern of staining. The nature of this staining pattern is uncertain; these structures may represent some sort of abortive tight junction formation or accumulation of degraded protein.

In summary, our findings indicate that claudin-4 expression is strongly associated with true epithelial differentiation and may be a useful marker to distinguish SWI/SNF complex-deficient undifferentiated carcinomas from sarcomas with epithelioid 
morphology. Whether it is clinically important to distinguish between these classes of aggressive neoplasms remains to be seen. The lack of claudin4 expression in ovarian small cell carcinoma of hypercalcemic type suggests that these tumors may be better regarded as sarcomas rather than carcinomas, but this needs further clarification.

\section{Disclosure/conflict of interest}

The authors declare no conflict of interest.

\section{References}

1 Gonzalez-Mariscal L, Betanzos A, Nava P, et al. Tight junction proteins. Prog Biophys Mol Biol 2003;81:1-44.

2 Morita K, Furuse M, Fujimoto K, et al. Claudin multigene family encoding four-transmembrane domain protein components of tight junction strands. Proc Natl Acad Sci USA 1999;96:511-516.

3 Soini Y, Kinnula V, Kahlos K, et al. Claudins in differential diagnosis between mesothelioma and metastatic adenocarcinoma of the pleura. J Clin Pathol 2006;59:250-254.

4 Ordonez NG. Value of claudin-4 immunostaining in the diagnosis of mesothelioma. Am J Clin Pathol 2013;139: 611-619.

5 Facchetti F, Lonardi S, Gentili F, et al. Claudin 4 identifies a wide spectrum of epithelial neoplasms and represents a very useful marker for carcinoma versus mesothelioma diagnosis in pleural and peritoneal biopsies and effusions. Virchows Arch 2007;451: 669-680.

6 Schuetz AN, Rubin BP, Goldblum JR, et al. Intercellular junctions in Ewing sarcoma/primitive neuroectodermal tumor: additional evidence of epithelial differentiation. Mod Pathol 2005;18:1403-1410.

7 Billings SD, Walsh SV, Fisher C, et al. Aberrant expression of tight junction-related proteins ZO-1, claudin-1 and occludin in synovial sarcoma: an immunohistochemical study with ultrastructural correlation. Mod Pathol 2004;17:141-149.

8 Kadoch C, Crabtree GR. Mammalian SWI/SNF chromatin remodeling complexes and cancer: mechanistic insights gained from human genomics. Sci Adv 2015;1: e1500447.

9 Agaimy A, Daum O, Markl B, et al. SWI/SNF complexdeficient undifferentiated/rhabdoid carcinomas of the gastrointestinal tract: a series of 13 cases highlighting mutually exclusive loss of SMARCA4 and SMARCA2 and frequent co-inactivation of SMARCB1 and SMARCA2. Am J Surg Pathol 2016;40:544-553.
10 Strehl JD, Wachter DL, Fiedler J, et al. Pattern of SMARCB1 (INI1) and SMARCA4 (BRG1) in poorly differentiated endometrioid adenocarcinoma of the uterus: analysis of a series with emphasis on a novel SMARCA4-deficient dedifferentiated rhabdoid variant. Ann Diagn Pathol 2015;19:198-202.

11 Agaimy A, Bertz S, Cheng L, et al. Loss of expression of the SWI/SNF complex is a frequent event in undifferentiated/dedifferentiated urothelial carcinoma of the urinary tract. Virchows Arch 2016;469:321-330.

12 Bell D, Hanna EY, Agaimy A, et al. Reappraisal of sinonasal undifferentiated carcinoma: SMARCB1 (INI1)deficient sinonasal carcinoma: a single-institution experience. Virchows Arch 2015;467:649-656.

13 Bishop JA, Antonescu CR, Westra WH. SMARCB1 (INI-1)-deficient carcinomas of the sinonasal tract. Am J Surg Pathol 2014;38:1282-1289.

14 Agaimy A, Koch M, Lell M, et al. SMARCB1(INI1)deficient sinonasal basaloid carcinoma: a novel member of the expanding family of SMARCB1-deficient neoplasms. Am J Surg Pathol 2014;38:1274-1281.

15 Peterson CL, Dingwall A, Scott MP. Five SWI/SNF gene products are components of a large multisubunit complex required for transcriptional enhancement. Proc Natl Acad Sci USA 1994;91:2905-2908.

16 Kadoch C, Hargreaves DC, Hodges C, et al. Proteomic and bioinformatic analysis of mammalian SWI/SNF complexes identifies extensive roles in human malignancy. Nat Genet 2013;45:592-601.

17 Hollmann TJ, Hornick JL. INI1-deficient tumors: diagnostic features and molecular genetics. Am J Surg Pathol 2011;35:e47-e63.

18 Sapi Z, Papp G, Szendroi M, et al. Epigenetic regulation of SMARCB1 By miR-206, -381 and -671-5p is evident in a variety of SMARCB1 immunonegative soft tissue sarcomas, while miR-765 appears specific for epithelioid sarcoma. A miRNA study of 223 soft tissue sarcomas. Genes Chromosomes Cancer 2016;55:786-802.

$19 \mathrm{Wu}$ JN, Roberts CW. ARID1A mutations in cancer: another epigenetic tumor suppressor? Cancer Discov 2013;3:35-43.

20 Karnezis AN, Wang Y, Ramos P, et al. Dual loss of the SWI/SNF complex ATPases SMARCA4/BRG1 and SMARCA2/BRM is highly sensitive and specific for small cell carcinoma of the ovary, hypercalcaemic type. J Pathol 2016;238:389-400.

21 Young RH, Oliva E, Scully RE. Small cell carcinoma of the ovary, hypercalcemic type. A clinicopathological analysis of 150 cases. Am J Surg Pathol 1994;18:1102-1116.

22 Clarke BA, Witkowski L, Ton Nu TN, et al. Loss of SMARCA4 (BRG1) protein expression as determined by immunohistochemistry in small-cell carcinoma of the ovary, hypercalcaemic type distinguishes these tumours from their mimics. Histopathology 2016;69: 727-738. 